International Journal of Pure and Applied Mathematics

Volume 93 No. 3 2014, 325-337

ISSN: 1311-8080 (printed version); ISSN: 1314-3395 (on-line version)

url: http://www.ijpam.eu

doi: http://dx.doi.org/10.12732/ijpam.v93i3.3

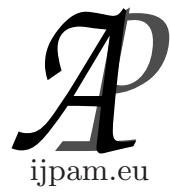

\title{
EXISTENCE THEOREM FOR AN INITIAL VALUE PROBLEM OF FRACTIONAL ORDER IN $L_{1}(0,1)$
}

\author{
Ibrahim Abouelfarag ${ }^{1}$, Asmaa M. Awad ${ }^{2}$ \\ ${ }^{1,2}$ Mathematics Department \\ Faculty of Science \\ Suez Canal University \\ Ismailia, 41522, EGYPT \\ ${ }^{1}$ Mathematics Department \\ Faculty of Science and Education \\ Taif University \\ Al-Khurmah Branch, KINGDOM OF SAUDI ARABIA
}

\begin{abstract}
In this paper we study the existence of solution $x \in L_{1}(0,1)$ for a functional integral equation. As an application we prove the existence of solution for an initial value problem of the fractional order. The main tools used are Schauder fixed point theorem, Lusin theorem and Scorza Dragoni theorem.
\end{abstract}

AMS Subject Classification: 45G10

Key Words: nonlinear functional integral equation, Fredholm operator, Volterra operator, fractional calculus

\section{Introduction}

The class of the integral equations, arises in several fields such as mathematical analysis, nonlinear functional analysis and mathematical physics [8],[9],[11],[15].

In this work, we use Schauder fixed point theorem and a set of conditions on $f_{1}, f_{2}, k_{1}, k_{2}$ and $g$ to prove the existence of $L_{1}$-solution of equation

Received: November 6, 2013

(C) 2014 Academic Publications, Ltd.

$\S_{\text {Correspondence author }}$ url: www.acadpubl.eu 


$$
x(t)=g(t)+\int_{0}^{1} k_{1}(t, s) f_{1}\left(s, f_{2}\left(s, \int_{0}^{s} k_{2}(s, \tau) x(\tau) d \tau\right)\right)^{d s, t \in(0,1) .}
$$

The set of conditions we imposed to prove the existence of $L_{1}$-solution of equation (1), turn to be naturally satisfied in some applications. In particular, we can apply our existence result to establish the existence of $L_{1}$-solution of the initial value problem of fractional order

$$
\begin{gathered}
\frac{d y(t)}{d t}=g(t)+\int_{0}^{1} k_{1}(t, s) f_{1}\left(s, f_{2}\left(s, D^{\beta} y(s)\right)\right) d s, \text { and } t>0 \\
y(0)=y_{0}
\end{gathered}
$$

where $D^{\beta} y(s)$ is the fractional derivative of $y$ and $\beta \in(0,1]$.

Sections 2,3 cover the relevant material needed in our work. The main result is given in Section 4. We discuss a special case (convolution type) of our integral equation (1) in Section 5. Finally, our application in initial value problem of fractional order is presented in Section 6 .

\section{Preliminaries}

In this section we recall some notations and results that will be needed to establish the main result.

Define $L_{p}=L_{p}(0,1), 1 \leq p<\infty$ as the set of all measurable functions on the interval $(0,1)$ where $|x|^{p}$ is Lebesgue integrable function on the interval $(0,1)$, for such function we define its norm

$$
\|x\|_{p}=\left\{\int_{0}^{1}|x(t)|^{p} d t\right\}^{\frac{1}{p}} .
$$

Definition 1. Assume that $f:(0,1) \times R \rightarrow R$ satisfies Carathéodory conditions, that is $f(t, x)$ is measurable in $t$ for any $x \in R$ and continuous in $x$ for almost all $t \in(0,1), x \in R$. Then to every function $x$ that is measurable on the interval $(0,1)$ we may assign the function

$$
(F x)(t)=f(t, x(t)), t \in(0,1)
$$

The operator $F$ defined in this way is called the superposition operator generated by the function $f$. 
We remark that this operator is one of the simplest and most important operators that is investigated in nonlinear functional analysis $[1],[2],[4],[5],[6],[7]$. Furthermore, we have the following theorem [10],[12].

Theorem 2. Let the function $f$ satisfy the conditions in Definition 1. The superposition operator $F$ generated by the function $f$ maps continuously the space $L_{1}(0,1)$ into itself if and only if

$$
|f(t, x)| \leq a(t)+b|x|,
$$

for all $t \in(0,1)$ and $x \in R$, where $a$ is a function belong to $L_{1}(0,1)$ and $b$ is a nonnegative constant.

Definition 3. Suppose $E$ is a real Banach space. A mapping $H: E \rightarrow E$ is said to be completely continuous if $H$ is continuous and $H(Y)$ is relatively compact for every bounded subset $Y$ of $E$.

The next theorems will be used to prove our result [16],[17],[18].

Theorem 4 (Schauder). Let $C$ be a nonempty, convex, closed, and bounded subset of a Banach space $E$. Let $H: C \rightarrow C$ be a completely continuous mapping. Then $H$ has at least one fixed point in $C$.

Theorem 5 (Lusin). Let $\varphi:[0,1] \rightarrow R$ be masurable function. for any $\epsilon>0$ there is a closed subset $A_{\epsilon}$ of $[0,1]$, with meas. $\left(A_{\epsilon}^{c}\right)<\epsilon$, such that $\left.\varphi\right|_{A_{\epsilon}}$ is continuous.

Theorem 6 (Scorza Dragoni). Let $f:[0,1] \times R \rightarrow R$ be a function satisfying Carathèodory hypothesis. Then for eash $\epsilon>0$ ther exists a closed subset $D_{\epsilon}$ of $[0,1]$, with meas. $\left([0,1]-D_{\epsilon}\right)=$ meas. $D_{\epsilon}^{c}<\epsilon$ and such that $\left.f\right|_{D_{\epsilon} \times R}$ is continuous.

\section{Fractional Calculus}

We give the definition of both differential and integral operator of fractional order. Furthermore, we give some results that are needed in the development of our main result [13],[14].

Definition 7. Let $f \in L_{1}[0,1]$ and $\beta \in R_{+}=[0, \infty)$. The RiemmanLiouville $(R-L)$ fractional order integral of the function $f$ of order $\beta$ is defined as

$$
I_{0}^{\beta} f(t)=\int_{0}^{t} \frac{(t-s)^{\beta-1}}{\Gamma(\beta)} f(s) d s, 0 \leq t \leq 1 .
$$


Definition 8. Let $g(t)$ be an absolutely continuous function on $[0,1]$. Then the fractional derivative of order $\alpha \in(0,1]$ of $g(t)$ is defined as

$$
D_{a}^{\alpha} g(t)=I_{a}^{1-\alpha} D g(t), \quad D=\frac{d}{d t} .
$$

Next, we list some basic properties of differential and integral operators of fractional order that will be used frequently in this work [5].

1. The operator $I_{a}^{\alpha}$ maps $L_{1}$ into itself continuously.

2. Let $f \in L_{1}$ and $\alpha, \beta \in(0,1]$, then $I_{a}^{\alpha} I_{a}^{\beta} f(t)=I_{a}^{\alpha+\beta} f(t)$. Furthermore, $\left(I_{a}^{\alpha}\right)^{n} f(t)=I_{a}^{n \alpha} f(t), n=1,2,3, \cdots$.

3. Let $\alpha, \beta \in(0,1]$ and $f, D f \in L_{1}$, we have $D I_{a}^{\alpha} f(t)=I_{a}^{\alpha} D f(t)$, when $f(a)=0$.

\section{Existence of Solution}

In this section, we present our main result by proving the existence of solutions of the nonlinear integral equation of mixed type (1) . Let us suppose the operator,

$$
T x(t)=g(t)+\int_{0}^{1} k_{1}(t, s) f_{1}\left(s, f_{2}\left(s, \int_{0}^{s} k_{2}(s, \tau) x(\tau) d \tau\right)\right)^{d s}
$$

First we impose the following conditions on the functions appearing in equation (3).

(i) $g \in L_{1}=L_{1}(0,1)$, a.e. nonnegative on $(0,1)$.

(ii) $f_{i}:(0,1) \times R \rightarrow R, i=1,2$ satisfy Caratheodory condition i.e $f$ is measurable in $t$ for any $x \in R$ and continuous in $x$ for almost all $t \in(0,1)$.

( iii) There are two functions $a_{i} \in L_{1}, i=1,2$ and two positive constants $b_{i}$, $i=1,2$ such that

$$
\left|f_{i}(t, x(t))\right| \leq a_{i}(t)+b_{i}|x|, \quad i=1,2
$$

for all $t \in(0,1)$. 
(iv) $k_{i}:(0,1) \times(0,1) \rightarrow R, i=1,2$ are measurable and the integral operators $K_{i}, i=1,2$ maps the space $L_{1}$ into itself.

$(v)$ The positive constant $c$ satisfies $c=b_{1} b_{2}\left\|K_{1}\right\|\left\|K_{2}\right\|<1$.

Now we have the following lemma which is needed in proving our main result.

Lemma 9. Let the above hypotheses $(i) \rightarrow(v)$ be satisfied. Then there exists a unique a.e. nonnegative function $x_{0}$ belonging to $L_{1}$, such that

$$
x_{0}(t)=g(t)+\int_{0}^{1} k_{1}(t, s)\left\{a_{1}(s)+b_{1}\left(a_{2}(s)+b_{2} \int_{0}^{s} k_{2}(s, \tau) x_{0}(\tau) d \tau\right)\right\} d s .
$$

Proof. Set

$$
\psi(t)=g(t)+\int_{0}^{1} k_{1}(t, s)\left\{a_{1}(s)+b_{1} a_{2}(s)\right\} d s
$$

clearly $\psi \in L_{1}$ according to condition (iv). Let $B_{r}=\left\{x: x \in L_{1},\|x\| \leq r\right\}$ where,

$$
r=\frac{\|\psi\|}{1-c}=\frac{\|\psi\|}{1-b_{1} b_{2}\left\|K_{1}\right\|\left\|K_{2}\right\|} .
$$

Next, we consider the operator $A$ that is defined by

$$
A x(t)=g(t)+\int_{0}^{1} k_{1}(t, s)\left\{a_{1}(s)+b_{1}\left(a_{2}(s)+b_{2} \int_{0}^{s} k_{2}(s, \tau) x(\tau) d \tau\right)\right\} d s
$$

clearly, the operator $A$ maps the space $L_{1}$ into itself . Moreover we can get, for $x \in B_{r}$

$$
\begin{aligned}
\|A x\| \leq & \int_{0}^{1}\left\{|g(t)|+\left|\int_{0}^{1} k_{1}(t, s)\left\{a_{1}(s)+b_{1} a_{2}(s)\right\} d s\right|\right\} d t+ \\
& +\int_{0}^{1}\left\{\int_{0}^{1} b_{1} b_{2}\left|k_{1}(t, s)\right|\left(\int_{0}^{s}\left|k_{2}(s, \tau)\right||x(\tau)| d \tau\right) d s\right\} d t \\
\leq & \|\psi\|+b_{1} b_{2}\left\|K_{1}\right\|\left\|K_{2}\right\|\|x\| \\
\leq & \|\psi\|+b_{1} b_{2}\left\|K_{1}\right\|\left\|K_{2}\right\| r
\end{aligned}
$$




$$
\leq\|\psi\|+b_{1} b_{2}\left\|K_{1}\right\|\left\|K_{2}\right\|\left(\frac{\|\psi\|}{1-b_{1} b_{2}\left\|K_{1}\right\|\left\|K_{2}\right\|}\right)=r
$$

This shows that $A$ maps $B_{r}$ into itself.

Next, let $Q_{r}$ be subset of $B_{r}$ consisting of all functions that are a.e. nonnegative on $(0,1), Q_{r}=\left\{x: x \in B_{r}, x(t) \geq 0\right.$ a.e. $\}$. Furthermore, $Q_{r}$ is closed subset of $L_{1}$ to show this, let us take $\left\{x_{n}\right\}$ be a convergent sequence of elements in $Q_{r}$ converging to $x$ ( in norm of $L_{1}(0,1),\left\|x_{n}-x\right\|_{L_{1}} \rightarrow 0$ ) this implies that the sequence $\left\{x_{n}\right\}$ converges in measure to $x$. Using Vitali theorem [19], we deduce that the sequence $\left\{x_{n}\right\}$ contains a subsequence $\left\{x_{n_{k}}\right\}$ that converges a.e. to $x$ on $(0,1)$. Since $\left\{x_{n}\right\}$ is nonnegative sequence, then its limit $x$ is also nonnegative on $(0,1)$. Which mean that $x \in Q_{r}$ and hence $Q_{r}$ is closed subset of $L_{1}$ and so it is a complete metric space.

Now, we prove that $\left.A\right|_{Q_{r}}$ is contraction mapping. Let $x_{1}, x_{2} \in Q_{r}$ we have,

$$
\begin{aligned}
\left\|A x_{1}-A x_{2}\right\| & =\int_{0}^{1}\left|A x_{1}(t)-A x_{2}(t)\right| d t \\
& \leq \int_{0}^{1} \int_{0}^{1}\left|k_{1}(t, s)\right|\left\{b_{1} b_{2} \int_{0}^{1}\left|k_{2}(s, \tau)\right| d \tau \int_{0}^{1}\left|x_{1}(\tau)-x_{2}(\tau)\right| d \tau\right\} d s d t \\
& \leq b_{1} b_{2}\left\|K_{1}\right\|\left\|K_{2}\right\|\left\|x_{1}-x_{2}\right\| .
\end{aligned}
$$

Since $0<b_{1} b_{2}\left\|K_{1}\right\|\left\|K_{2}\right\|<1$, then we deduce that $\left.A\right|_{Q_{r}}$ is contraction mapping. Then there exists a unique a.e. nonnegative function $x_{0}$ belonging to $L_{1}$ that satisfied equation (4)

Theorem 10. Assume that the conditions $(i) \rightarrow(v)$ are satisfied and $k$ satisfies the Carathéodory condition. Then the equation (1) has at least one solution $x \in L_{1}$.

Proof. We have two cases.

First, assume that $x_{0}$ the zero element of $L_{1}$ solves equation (4) and let,

$$
y(t)=g(t)+\int_{0}^{1} k_{1}(t, s) f_{1}\left(s, f_{2}\left(s, \int_{0}^{s} k_{2}(s, \tau) x_{0}(\tau) d \tau\right)\right)^{d s \quad t \in(0,1)}
$$

then from condition (iii) we get, $|y(t)| \leq|g(t)|+\int_{0}^{1}\left|k_{1}(t, s)\right|\left\{\left|a_{1}(s)+b_{1}\left(a_{2}(s)+b_{2} \int_{0}^{s} k_{2}(s, \tau) x_{0}(\tau) d \tau\right)\right|\right\} d s$. 
$|y(t)| \leq x_{0}(t), \quad$ a.e.

From Lemma 9, we get $y=0$ and hence $x_{0}$ solve equation (1).

Second, we assume that $x_{0}$ is not the zero element of $L_{1}$ and we consider the set $Q=\left\{y: y \in L_{1},|y(t)| \leq x_{0}(t)\right.$,a.e $\}$ This set $Q$ can be shown to be nonempty, closed, bounded and convex in $L_{1}$.

Now let be the nonlinear operator associated with equation (1) and defined by

$T x(t)=g(t)+\int_{0}^{1} k_{1}(t, s) f_{1}\left(s, f_{2}\left(s, \int_{0}^{s} k_{2}(s, \tau) x(\tau) d \tau\right)\right)^{d s, \quad t \in(0,1) .}$

Based on our assumptions, we can see that $T$ maps $L_{1}$ into itself continuously, we will prove that $T: Q \rightarrow Q$. Let $x \in Q$ then

$$
\begin{aligned}
|T x| & =\left|g(t)+\int_{0}^{1} k_{1}(t, s) f_{1}\left(s, f_{2}\left(s, \int_{0}^{s} k_{2}(s, \tau) x(\tau) d \tau\right)\right\} d s\right| \\
& \leq|g(t)|+\int_{0}^{1}\left|k_{1}(t, s)\right|\left\{\left|a_{1}(s)\right|+b_{1}\left(\left|a_{2}(s)\right|+b_{2} \int_{0}^{s}\left|k_{2}(s, \tau)\right|\left|x_{0}(\tau)\right| d \tau\right)\right\} d s \\
|T x| & \leq x_{0}(t), \text { a.e. }
\end{aligned}
$$

Hence $T x$ belong to $Q$.

In the following we show that the set $T(Q)$ is relatively compact in $L_{1}$. So the Schauder fixed point principle works to get our aim. To achieve this we assume that $\left\{y_{n}\right\}$ is a sequence in $Q$ and fixed $\epsilon>0$. Upon using Theorem 5 and Theorem 6 imply that there is a closed subset $D_{\epsilon}$ of $[0,1]$ with means. $D_{\epsilon}^{c}<\epsilon\left(D_{\epsilon}^{c}=[0,1]-D_{\epsilon}\right)$ such that all the restriction $\left.g\right|_{D_{\epsilon}},\left.x_{0}\right|_{D_{\epsilon}}$ and $\left.k_{1}\right|_{D_{\epsilon} \times[0,1]},\left.k_{2}\right|_{D_{\epsilon} \times[0,1]}$ are uniformly continuous. In what follows we show that $\left\{y_{n}\right\}$ is equicontinuous on $D_{\epsilon}$, for that let $\epsilon_{1}>0$ be given since $g$ is uniformly continuous on $D_{\epsilon}$, there exists $\delta>0$ such that $t^{\prime}, t^{\prime \prime} \in D_{\epsilon},\left|t^{\prime \prime}-t^{\prime}\right|<\delta$ implies that

$$
\left|g\left(t^{\prime \prime}\right)-g\left(t^{\prime}\right)\right|<\frac{\epsilon_{1}}{2}
$$

also $\left.k_{1}\right|_{D_{\epsilon} \times[0,1]}$ is uniformly continuous, there exists $\delta>0$ such that

$t^{\prime}, t^{\prime \prime} \in D_{\epsilon},\left|t^{\prime \prime}-t^{\prime}\right|<\delta$ we have

$$
\left|k_{1}\left(t^{\prime \prime}, s\right)-k_{1}\left(t^{\prime}, s\right)\right|<\frac{\epsilon_{1}}{2 \gamma}
$$


where

$$
\gamma=\left\|a_{1}\right\|+b_{1}\left\|a_{2}\right\|+b_{1} b_{2}\left\|K_{2}\right\|\left\|x_{0}\right\|
$$

Finally, for $t^{\prime}, t^{\prime \prime} \in D_{\epsilon},\left|t^{\prime \prime}-t^{\prime}\right|<\delta$ we get

$$
\begin{aligned}
&\left|T y_{n}\left(t^{\prime \prime}\right)-T y_{n}\left(t^{\prime}\right)\right| \leq\left|g\left(t^{\prime \prime}\right)-g\left(t^{\prime}\right)\right|+\int_{0}^{1}\left|k_{1}\left(t^{\prime \prime}, s\right)-k_{1}\left(t^{\prime}, s\right)\right| . \\
&\left|\left\{a_{1}(s)+b_{1}\left(a_{2}(s)+b_{2} \int_{0}^{s} k_{2}(s, \tau) x_{0}(\tau) d \tau\right)\right\}\right| d s \\
& \leq \frac{\epsilon_{1}}{2}+\frac{\epsilon_{1}}{2 \gamma} \gamma=\epsilon_{1} .
\end{aligned}
$$

Also, the sequence $\left\{T\left(y_{n}\right)\right\}$ is a sequence of uniformly bounded function, since

$$
\begin{aligned}
\left|T y_{n}(t)\right| \leq & |g(t)|+ \\
& +\int_{0}^{1}\left|k_{1}(t, s)\right|\left\{\left|a_{1}(s)\right|+b_{1}\left(\left|a_{2}(s)\right|+b_{2} \int_{0}^{s}\left|k_{2}(s, \tau)\right|\left|x_{0}(\tau)\right| d \tau\right)\right\} d s \\
\leq & x_{0}(t), \text { a.e. }
\end{aligned}
$$

Hence

$$
\left\|T\left(y_{n}\right)\right\|_{C\left(D_{\epsilon}\right)} \leq\left\|x_{0}\right\|_{C\left(D_{\epsilon}\right)}, \forall n .
$$

This implies that the sequence $\left\{T\left(y_{n}\right)\right\}$ is a sequence of uniformly bounded and equicontinuous functions on $D_{\epsilon}$. Hence, in view of Ascoli-Arzĕla theorem [3] we deduce that $\left\{T\left(y_{n}\right)\right\}$ is relatively compact subset of $C\left(D_{\epsilon}\right)$. Now, let us observe that the above proof does not depend on the choice of $\epsilon$. Thus we construct a sequence $\left\{D_{k}\right\}$ of closed subsets of $[0,1]$ with meas. $D_{k}^{c} \rightarrow 0$ such that $\left\{T\left(y_{n}\right)\right\}$ is relatively compact subset of $C\left(D_{k}\right)$. This implies that $\left\{T\left(y_{n}\right)\right\}$ has a convergent subsequence in each $C\left(D_{k}\right), k=1,2 \ldots$ But $C\left(D_{k}\right)$ is a complete metric space, hence this subsequence $\left\{T\left(y_{n}\right)\right\}$ is Cauchy sequence in each $C\left(D_{k}\right), k=1,2, .$. , for any $\epsilon>0$, and $n, m$ arbitrary large $\left\|T y_{m}-T y_{n}\right\|_{C\left(D_{k}\right)}<\epsilon$

But we want to prove that the set $T(Q)$ is relatively compact in $L_{1}$ that is $\overline{T(Q)}$ is compact in $L_{1}$. To do this, we will prove that the sequence $\left\{T\left(y_{n}\right)\right\}$ is convergent sequence in $L_{1}$. Since $L_{1}$ is complete metric space, then it is sufficient to prove that the sequence $\left\{T\left(y_{n}\right)\right\}$ is Cauchy sequence in $L_{1}$.

Let $\eta>0$ be given,we will show that there exists $N(\eta)$ such that 
$\left\|T y_{m}-T y_{n}\right\|_{L_{1}}<\eta$, whenever $n, m>N(\eta)$. Given $\eta>0$, there is a $\delta>0$ such that meas. $D_{\delta}<\delta$ implies

$$
\int_{D_{\delta}} x_{0}(s) d s<\frac{\eta}{4}
$$

Choose $k^{*} \in \mathbb{N}$ with meas. $D_{k^{*}}^{c}<\delta$ and

$$
\begin{aligned}
\int_{0}^{1}\left|\left(T y_{m}\right)(t)-\left(T y_{n}\right)(t)\right| d t & =\int_{D_{k^{*}}^{c}}\left|\left(T y_{m}\right)(t)-\left(T y_{n}\right)(t)\right| d t+ \\
& +\int_{D_{k^{*}}}\left|\left(T y_{m}\right)(t)-\left(T y_{n}\right)(t)\right| d t \\
& \leq \int_{D_{k^{*}}}\left|\left(T y_{m}\right)(t)\right|+\left|\left(T y_{n}\right)(t)\right| d t+ \\
& +\left\|T y_{m}-T y_{n}\right\|_{C\left(D_{k^{*}}^{c}\right)} \int_{D_{k^{*}}} d t \\
\int_{0}^{1}\left|\left(T y_{m}\right)(t)-\left(T y_{n}\right)(t)\right| d t \leq & \frac{\eta}{4}+\frac{\eta}{4}+\left\|T y_{m}-T y_{n}\right\|_{C\left(D_{k^{*}}^{c}\right)}(1-\delta) \\
\leq & \frac{\eta}{2}+\left\|T y_{m}-T y_{n}\right\|_{C\left(D_{k^{*}}^{c}\right)} \cdot
\end{aligned}
$$

Choose $N$ such that $n, m>N$ implies $\left\|T y_{m}-T y_{n}\right\|_{C\left(D_{k^{*}}\right)}<\frac{\eta}{2}$. Then

$$
\int_{0}^{1}\left|\left(T y_{m}\right)(t)-\left(T y_{n}\right)(t)\right| d t=\frac{\eta}{2}+\frac{\eta}{2}=\eta
$$

This means that $\left\{T\left(y_{n}\right)\right\}$ is a Cauchy a sequence in the space $L_{1}$ and hence the set $T(Q)$ is relatively compact in $L_{1}$. Then $T$ has at least one fixed point. Hence there exists at least one solution $x \in L_{1}$ of equation (1). 


\section{Convolution Type Integral Equation}

Assume that $k:(0,1) \rightarrow R_{+}$is an measurable function. For an arbitrary function $x \in L_{1}$ put

$$
(K x)(t)=\int_{0}^{t} k(t-s) x(s) d s, \quad t \in(0,1) .
$$

This operator $K$ is a linear integral operator of convolution type and maps $L_{1}$ into itself continuously

Now consider the following condition

$$
\text { (vi) } k:(0,1) \rightarrow R_{+} \text {and } k \in L_{1} \text {. }
$$

Then we have the following corollary

Corollary 11. Let the hypotheses $(i) \rightarrow(v i)$ be satisfied. Then there exists a unique a.e. nonnegative function $x_{0}$ belonging to $L_{1}$ such that

$x_{0}(t)=g(t)+\int_{0}^{1} k_{1}(t, s)\left\{a_{1}(s)+b_{1}\left(a_{2}(s)+b_{2} \int_{0}^{s} k_{2}(s-\tau)\left(a_{2}(\tau)+x_{0}(\tau)\right) d \tau\right\} d s\right.$.

Furthermore, the equation

$$
x(t)=g(t)+\int_{0}^{1} k_{1}(t, s) f_{1}\left(s, f_{2}\left(s, \int_{0}^{s} k_{2}(s-\tau) x(\tau) d \tau\right)\right) d s, \quad t \in(0,1)
$$

has at least one integrable solution $x \in L_{1}$.

\section{Initial Value Problems of Fractional Order}

As a special case of equation (5), we consider

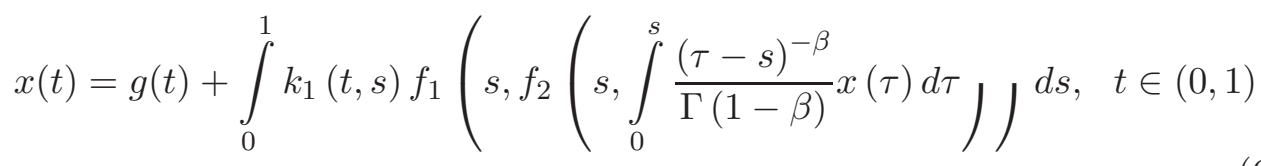


where $k_{2}(\tau-s)=\frac{(\tau-s)^{-\beta}}{\Gamma(1-\beta)}$. Equation (6) is an integral equation of fractional order that can be written in the form

$$
x(t)=g(t)+\int_{0}^{1} k_{1}(t, s) f_{1}\left(s, f_{2}\left(s, I^{1-\beta} x(s)\right)\right) d s .
$$

Clearly, according to Corollary 11, equation (7) has at least one solution $x \in L_{1}$.

Definition 12. By a solution of the initial value problem (2) we mean an absolutely continuous function $x$ satisfies the initial value problem (2).

Theorem 13. Let $\beta \in(0,1]$. Let $k_{1}$ and $f_{1}$ satisfy the conditions of Theorem 10. If $c=b_{1}\left\|K_{1}\right\|<1$, then the initial value problem (2) has at least one solution $x \in L_{1}$

Proof. Let $x$ be a solution of equation (7). Putting,

$$
y(t)=\int_{0}^{t} x(\tau) d \tau
$$

Since $x$ is integrable on $(0,1)$, then,

$$
D y(t)=D \int_{0}^{t} x(\tau) d \tau \quad \text { a.e. }
$$

where $D=\frac{d}{d t}$.

Moreover, the integral $\int_{0}^{t} x(\tau) d \tau$ of integrable function $x$ is absolutely continuous then,

$$
D y(t)=D I_{0}^{1} x(t)
$$

Then we have,

$$
D y(t)=x(t) \quad \text { a.e. }
$$

Furthermore, we have,

$$
\begin{aligned}
I^{1-\beta} D y(t) & =I^{1-\beta} x(t) \\
D^{\beta} y(t) & =I^{1-\beta} x(t) .
\end{aligned}
$$


Consequently, we have,

$$
\frac{d y(t)}{d t}=D y(t)=g(t)+\int_{0}^{1} k_{1}(t, s) f_{1}\left(s, f_{2}\left(s, D^{\beta} y(s)\right)\right) d s .
$$

Since $x$ is integrable and absolutely continuous, then

$$
\begin{aligned}
D y(\tau) & =x(\tau) \\
\int_{0}^{t} \frac{d}{d t} y(\tau) d \tau & =\int_{0}^{t} x(\tau) d \tau \\
y(t)-y_{0} & =\int_{0}^{t} x(\tau) d \tau .
\end{aligned}
$$

Clearly, $y(0)=y_{0}$.

We deduced that this $y$ is an absolutely continuous function satisfies the initial value problem (2). Hence our proof is complete.

\section{References}

[1] J. Banas, On the superposition operator and integrable solutions of some functional equation, Nonlinear Anal. 12, No 8 (1988), 777784.http://dx.doi.org/10.1016/0362-546X(88)90038-7.

[2] M. Cichoń, A.M.A. El-Sayed and A.H. Hussien, Existence theorem for nonlinear functional integral equations of fractional orders, Comment. Math. Prace Matem 41, No 1 (2001), 59-67.

[3] N. Dunford and J. Schwartz, Linear Operator I, Int. Publ., Leyden, (1963).

[4] A.M.A. El-Sayed, Nonlinear functional differential equations of arbitrary orders, Nonlinear Anal. 33, No 2 (1998), 181-186. http://dx.doi.org/10.1016/S0362-546X(97)00525-7.

[5] A.M.A. El-Sayed, W.G. El-Sayed and O.L. Moustafa, On some fractional functional equations, Pure Math. Appl. 6, No 4 (1995), 321-332.

[6] A. M. A. El-Sayed, N. Sherif and I. A. Ibrahim, On a mixed-type integral equation and fractional-order functional differential equations, Comment. Math. Prace Mat., vol. 45, no. 2, (2005), 237-247 . 
[7] G. Emmanuele, Integrable solutions of a functional-integral equation, Integral Equations Appl. 4, No 1 (1992), 89-94.

[8] I. A. Ibrahim, Solvability of mixed type operator equations, Appl. Math. Comput., vol. 190 (2007), 1344-1352 .

[9] I. A. Ibrahim, On the existence of solutions of functional integral equation of Urysohn type, Comput. Math. Appl., vol. 57, no. 10, (2009),1609-1614 .

[10] M. A. Krasnoselskii, Topological Methods in the Theory of Nonlinear Integral Equations, Pergamon Press, Oxford, (1964).

[11] M. A. Krasnoselskii, P. P. Zabrejko, J. I. Pustylnik and P. J. Sobolevskii, Integral Operators in Spaces of Summable Functions ( Nauka, Moscow, 1966), [English Translation: Noordhoff, Leyden, 1976].

[12] M. Meehan and D. O'Regan, Positive $L^{p}$ solutions of Fredholm integral equations, Archiv der Mathematik 76 (2001), 366-376.

[13] K. S. Miller and B. Ross, An Introduction to Fractional Calculus and Fractional Differential Equations, John Wiley and Sons, New York,(1993).

[14] I. Podlubny and A. M. A. El-Sayed, On two definitions of fractional derivative, Slovak Academy of Sciences Institute of Experimental physics, UEF -03 - 96 ISBN 80-7099-252-2, (1996).

[15] P. P. Zabrejko, A. I. Koshelev, M. A. Krasnoselskii, S. G. Mikhlin, L. S. Rakovshchik, and V. J. Stecenko, Integral equations, Noordhoff, Leyden, (1975).

[16] Goebel, K. and Kirk, W. A., Topics in Metric Fixed Point Theory. Cambrige University Press, Cambrige, 1990.

[17] Dinculeanu, D., Vector Measures, Pergamon Press, (1967).

[18] Scorza Dragoni, G., Un teorema sulle funzioni continue rispetto ad une e misurabili rispetoo ad un'altra variable, Rend. Sem. Mat. Uni. Padova 17,(1948) 102-106.

[19] Natanson, I. P., Theory of Functions of Real Variable. Undar, New York,(1960). 
